\title{
O manejo multidisciplinar do paciente com demência frontotemporal à luz da literatura mundial
}

\author{
The multidisciplinary management of the patient with frontotemporal dementia in the light \\ of the world literature \\ El manejo multidisciplinario del paciente con demencia frontotemporal a la luz de la \\ literatura mundial
}

Nickolas Souza Silva ${ }^{1 *}$, Lady Jane da Silva Macedo², Alexandre Vitor Dias Silveira ${ }^{3}$, Bruna Araújo Rossi $^{4}$, José Leandro da Silva Menezes Diniz ${ }^{5}$, Mariana Gomes Santana ${ }^{5}$, Pedro Henrique de Souza $^{6}$, Pricilla Helena Sacavem ${ }^{7}$, Paulo César Monteiro Florêncio ${ }^{8}$, Larissa Teles de Souza ${ }^{8}$.

\section{RESUMO}

Objetivo: Analisar as principais formas de manejo multidisciplinar da demência frontotemporal na contemporaneidade. Métodos: Trata-se de uma revisão de literatura com busca bibliográfica realizada nas plataformas PUBMED, LILACS e SciELO. Foram utilizados os descritores "Demência Frontotemporal" e "Multidisciplinaridade". Foram critérios de inclusão artigos publicados entre 2010 e 2020. Resultados: Para escolha do tratamento da demência frontotemporal deve-se avaliar os hábitos e o ambiente do paciente. A prática de atividades físicas leves, como caminhadas, musicoterapia e psicoterapia apresentaram resultados positivos na diminuição da sintomatologia. Apesar de psicoterapia, musicoterapia e incentivo a atividades físicas leves serem as principais abordagens utilizadas, outras condutas também devem ser incentivadas. A linguagem é um dos pontos que deve ser avaliado, já que é útil na monitorização dos pacientes e de seu tratamento. $O$ tratamento multidisciplinar deve ser estendido para familiares e cuidadores das pessoas com demência frontotemporal. O programa de atividades personalizadas, que é baseado na terapia ocupacional, é uma das melhores formas de abordagem. Considerações Finais: Abordagens não farmacológicas, como psicoterapia, incentivo à prática de atividades físicas e musicoterapia são formas possíveis, atualmente, de cuidar dos pacientes com demência frontotemporal e de seus familiares.

Palavras-chave: Demência frontotemporal, Equipe de assistência ao paciente, Terapêutica.

\begin{abstract}
Objective: To analyze the main forms of multidisciplinary management of frontotemporal dementia in contemporary times. Methods: This is a literature review with bibliographic search performed on the platforms PUBMED, LILACS and SciELO. The descriptors "Frontotemporal Dementia" and "Multidisciplinarity" were used. Inclusion criteria were articles published between 2010 and 2020. Results: To choose the treatment of frontotemporal dementia the patient's habits and environment should be evaluated. The practice of light physical activities, such as walking, music therapy and psychotherapy showed positive results in reducing symptoms. Although psychotherapy, music therapy and encouragement of light physical activities are the main approaches used, other approaches should also be encouraged. Language is one of the points that must be evaluated, as it is useful in monitoring patients and their treatment. Multidisciplinary treatment should be extended to family members and caregivers of people with frontotemporal dementia. The personalized activities program, which is based on occupational therapy, is one of the best approaches. Final Considerations: Non-pharmacological approaches, such as psychotherapy, encouraging the practice of physical activities and music therapy, are currently the possibles ways to care for patients with frontotemporal dementia and their families.
\end{abstract}

Keywords: Frontotemporal dementia, Patient care team, Therapeutics.

\footnotetext{
1 Universidade Federal do Ceará, Sobral - CE. *E-mail: nickolassouza23@gmail.com

2 Instituto de Educação Superior do Vale do Parnaíba, Parnaíba - PI.

${ }^{3}$ Centro universitário FIPMoc, Montes Claros - MG.

4 Universidade Federal do Rio de Janeiro, Rio de Janeiro - RJ.

${ }^{5}$ Universidade Católica de Pernambuco, Recife - PE.

6 Universidade Estadual do Mato Grosso do Sul, Dourados - MS.

7 Universidade do Vale do Itajaí, Itajaí - SC.

8 Universidade Federal do Delta do Parnaíba, Parnaíba - PI.
} 


\section{RESUMEN}

Objetivo: Analizar las principales formas de manejo multidisciplinario de la demencia frontotemporal em los tempos contemporáneos. Métodos: Revisión bibliográfica con búsqueda bibliográfica realizada em las plataformas PUBMED, LILACS y SciELO. Se utilizaron los descriptores "Demencia frontotemporal" y "Multidisciplinariedad". Los criterios de inclusión fueron artículos publicados entre 2010 y 2020 . Resultados: para elegir el tratamiento de la demencia frontotemporal, se deben evaluarlos hábitos y el entorno del paciente. La práctica de actividades físicas ligeras, como caminar, musicoterapia y psicoterapia mostró resultados positivos en la reducción de los síntomas. Aunque la psicoterapia, la musicoterapia y el estímulo a las actividades físicas ligeras son los principales enfoques utilizados, también deben fomentar se otros enfoques. El lenguaje es uno de los puntos que deben evaluarse, ya que es útil para controlar a los pacientes y su tratamiento. El tratamento multidisciplinario debe extenderse a los familiares y cuidadores de personas con demencia frontotemporal. El programa de actividades personalizadas, que se basa en la terapia ocupacional, es uno de los mejores enfoques. Consideraciones Finales: Los enfoques no farmacológicos, como psicoterapia, que fomentan la práctica de actividades físicas y la musicoterapia, son actualmente las posibles formas de atender a los pacientes con demencia frontotemporal y sus familias.

Palabras clave: Demencia frontotemporal, Grupo de atención al paciente, Terapéutica.

\section{INTRODUÇÃO}

A Demência Frontotemporal (DFT) consiste em uma síndrome neurodegenerativa que afeta os lobos frontal e temporal, causando sintomatologia variada, conforme a área neuroanatômica acometida (CUSTÓDIO N, et al., 2012; PANTOJA G, et al., 2020). Em 1892, foram publicadas as primeiras descrições na literatura sobre casos de deterioração cognitiva, especialmente da linguagem, relacionados a atrofia focal ou circunscrita aos lobos temporais e frontais, pois pensavam que o processo degenerativo estaria disseminado em todo o encéfalo. Ao longo dos anos, as pessoas com DFT tinham seu diagnóstico associado aos portadores da Doença de Alzheimer (DA). Na década de 90 foram propostos critérios clínicos e neuropatológicos específicos para o diagnóstico correto de DFT (SOUZA MF, 2011; VALENTE ES, 2018; RODRÍGUES-LEYVA I, 2019).

O início da doença costuma ser mais precoce, em torno da $5^{\text {a }}$ década de vida (FORNARI LHT, et al., 2010). A expectativa de vida das pessoas que sofrem com a DFT é de 3 a 12 anos após o ano do diagnóstico, sendo comparável à estimativa da $D A$, o que está relacionado à falta de um tratamento definitivo (SIERRA-PELÁEZ S, VILLEGAS-LANAU CA, 2015).

No Brasil, não há um consenso ou estimativa bem pautada acerca da prevalência da DFT, mas estudos indicam uma variação de 12 a 20\% entre as demências (FREIRE R, 2014; LILLO P e LEYTON C, 2016). A relação hereditária da DFT é vista em $50 \%$ dos casos e destes em $10 \%$ ocorre herança dominante autossômica, apontando uma importante função dos elementos genéticos no aumento da DFT (CORDEIRO Q, et al., 2018).

O diagnóstico padrão-ouro é realizado por meio do exame neuropatológico, associado ao quadro clínico, marcadores bioquímicos e neuroimagem típica. A investigação é ampla, devido à dificuldade de diferenciar os sinais e os sintomas. A utilização de métodos de neuroimagem funcional e estrutural, técnicas de biologia molecular e genética molecular ajudam ao diagnóstico diferencial precoce das demências, antecipando o tratamento e melhorando o prognóstico (LILLO P e LEYTON C, 2016).

A terapêutica da DFT, na literatura médica, não possui relatos de um tratamento específico para interromper a progressão da doença e em parte dos casos é utilizada a abordagem farmacológica. Contudo, deve-se sempre estar associada ao tratamento não farmacológico, como as terapias linguística e fisioterápica, bem como, outras formas de tratamento multidisciplinar. A fim de garantir uma boa qualidade de vida e o conforto do paciente e seus cuidadores, é fundamental que o paciente, pratique exercícios físicos e tenha uma dieta equilibrada, visando a um melhor prognóstico global (O'CONNOR CM, et al., 2013).

O presente estudo tem como objetivo realizar uma revisão de literatura analisando a importância e os meios do tratamento multidisciplinar nos pacientes com DFT baseado na literatura mundial disponível nas plataformas de dados utilizadas. 


\section{MÉTODOS}

O presente artigo se estrutura como uma revisão de literatura, usando como base de dados as plataformas de busca Scientific Electronic Library Online(SciELO, do português "Livraria Científica Eletrônica Online"); Latin American and Caribbean Health Sciences Literature (Lilacs, do português "Literatura de Ciências da Saúde Latinoamericana e Caribenha"); e PubMed/MEDLINE, pautando-se nos descritores "Frontotemporal dementia" e "Multidisciplinary" sendo utilizados em português e inglês, nas plataformas supracitadas. Os descritores foram utilizados em combinação, usando o operador booleano aditivo restritivo na plataforma PubMed/MEDLINE. Para SciELO e Lilacs foi utilizado apenas o descritor "Frontotemporal dementia", devido à restrição de achados primários.

Os artigos foram selecionados a partir de critérios de inclusão, dentre os quais foram escolhidos a data de publicação, as línguas em que os artigos foram escritos, bem como, o tipo de publicação. Baseado nessa premissa foram selecionados os artigos publicados entre os anos 2010 a 2020, escritos em línguas portuguesa, inglesa e/ou espanhola. Foram, ainda, selecionadas publicações de artigos de revisão, relatos de casos e meta-análises, ao passo que foram adotadas como critérios de exclusão artigos de monografias, notas técnicas, capítulos de livro, resumos de eventos, editoriais, artigos de opinião e publicações anteriores ao ano de 2010, sendo então excluídas no processo primário de busca.

A abordagem multidisciplinar e o manejo foram selecionados como tópicos norteadores da elaboração deste presente estudo. A análise dos artigos, baseando-se nos pontos norteadores, foi avaliada em pares, considerando-se os critérios de inclusão e exclusão. A seleção dos artigos foi feita segundo a avaliação do título, seguido do resumo, e, por fim, da avaliação completa do artigo. O estudo foi realizado durante os meses de maio a julho de 2020 .

O resultado conta com a análise dos dados documentais dos 273 artigos encontrados na busca primária nas plataformas de buscas e sendo aplicados todos os critérios de inclusão e exclusão, sendo selecionados 30 artigos para composição do presente artigo (Figura 1).

Figura 1 - Fluxograma da metodologia de busca dos estudos.

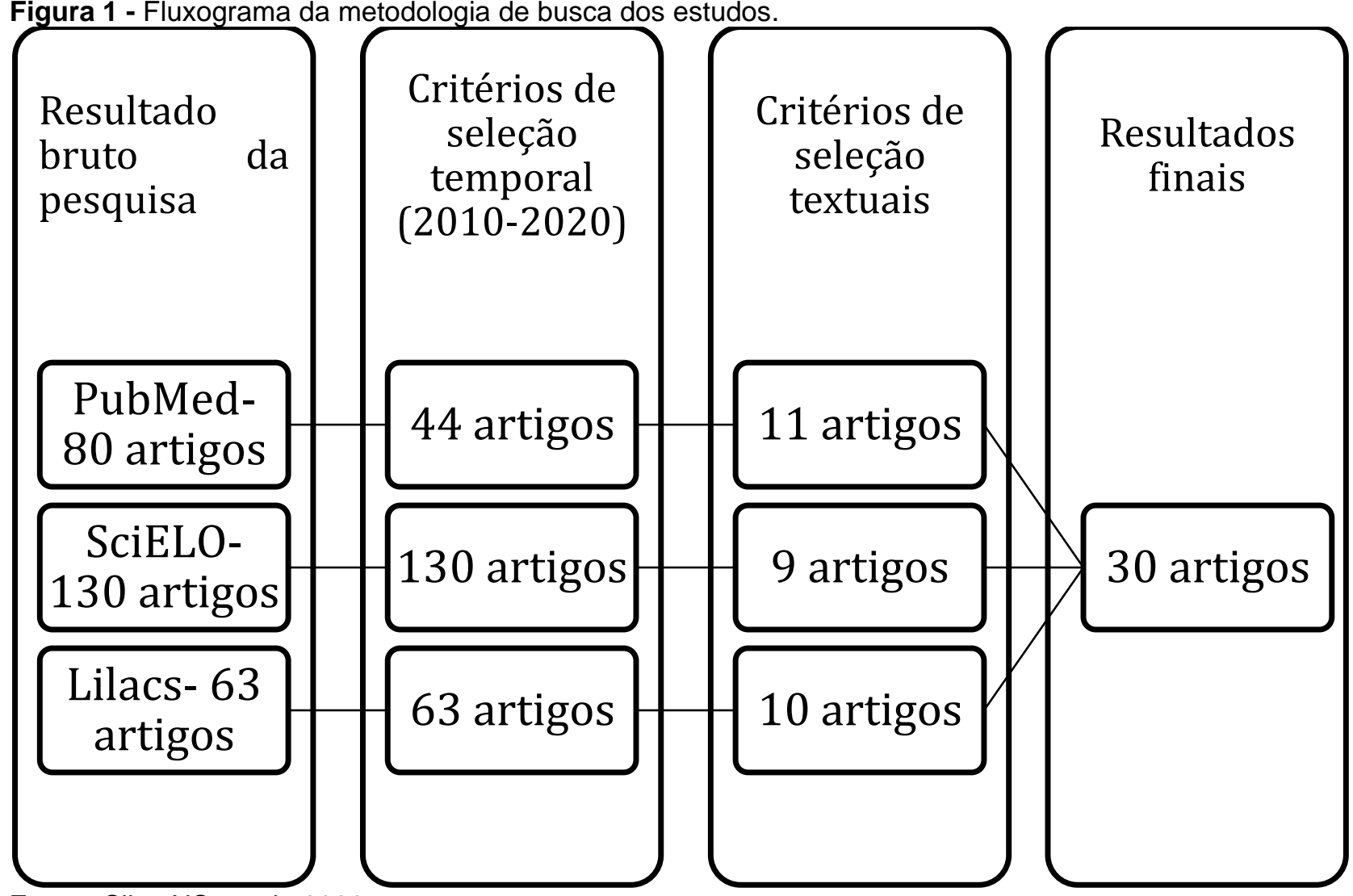

Fonte: Silva NS, et al., 2020. 


\section{RESULTADOS}

Acerca dos estudos analisados foi construída a discussão entre a DFT e suas variantes, a análise da terapêutica multidisciplinar atualmente instituída nesses pacientes, bem como, a funcionalidade das terapias multidisciplinares. Os estudos acerca da DFT e seus aspectos terapêuticos multidisciplinares foram debatidos e esquematizados segundo os tópicos abaixo descritos.

Existem inúmeras variantes de DFT, cujas manifestações clínicas e histopatológicas diferem amplamente entre si e são diferenciadas de acordo com a ordem de aparecimento dos sintomas ou com o sintoma predominante na clínica do paciente (Tabela 1) (SIERRA-PELÁEZ S, VILLEGAS-LANAU CA, 2015).

Quadro 1 - Variantes da demência frontotemporal.

\begin{tabular}{|c|c|c|c|}
\hline $\begin{array}{l}\text { Variante da demência } \\
\text { frontotemporal }\end{array}$ & Sintomatologia & Terapêutica & Marcador clínico \\
\hline $\begin{array}{c}\text { Demência } \\
\text { Frontotemporal } \\
\text { Variante } \\
\text { Comportamental }\end{array}$ & $\begin{array}{l}\text { Perda de habilidades } \\
\text { sociais/executivas, } \\
\text { perda da resposta } \\
\text { emocional, obsessão, } \\
\text { desinibição e mudanças } \\
\text { dos padrões alimentares }\end{array}$ & $\begin{array}{l}\text { Neurolépticos atípicos, } \\
\text { antidepressivos, } \\
\text { anticonvulsivantes, } \\
\text { agonistas da dopamina } \\
\text { e educação de } \\
\text { pacientes e cuidadores }\end{array}$ & $\begin{array}{c}\text { Memória visual e verbal } \\
\text { mais preservada em } \\
\text { relação a pacientes com } \\
\text { Demência de Alzheimer, } \\
\text { maior comprometimento } \\
\text { da função executiva e } \\
\text { da atenção }\end{array}$ \\
\hline $\begin{array}{c}\text { Demência } \\
\text { Frontotemporal Não } \\
\text { Fluente }\end{array}$ & $\begin{array}{c}\text { Fluência verbal } \\
\text { inadequada, parafasias } \\
\text { fonêmicas, apraxia da } \\
\text { fala, rigidez, } \\
\text { bradicinesia, disartria }\end{array}$ & $\begin{array}{l}\text { Agonistas de dopamina } \\
\text { e educação de } \\
\text { pacientes e cuidadores }\end{array}$ & $\begin{array}{l}\text { Pacientes com controle } \\
\text { executivo deficiente e } \\
\text { baixa memória } \\
\text { operacional }\end{array}$ \\
\hline $\begin{array}{l}\text { Demência } \\
\text { Frontotemporal } \\
\text { Semântica }\end{array}$ & $\begin{array}{c}\text { Modificações de } \\
\text { memória semântica, } \\
\text { perda de vocabulário, } \\
\text { anomia, redução da } \\
\text { compreensão de } \\
\text { palavras, } \\
\text { prosopagnosia, agnosia } \\
\text { visual, depressão, } \\
\text { desinibição, apatia, } \\
\text { alterações de } \\
\text { preferência alimentar }\end{array}$ & $\begin{array}{l}\text { Agonistas de dopamina } \\
\text { e educação de } \\
\text { pacientes e cuidadores }\end{array}$ & $\begin{array}{l}\text { Pacientes com maior } \\
\text { perda de memória } \\
\text { semântica e anomia em } \\
\text { relação aos outros tipos } \\
\text { de demência } \\
\text { frontotemporal }\end{array}$ \\
\hline $\begin{array}{l}\text { Demência } \\
\text { Frontotemporal } \\
\text { Logopênica }\end{array}$ & $\begin{array}{l}\text { Relativa preservação da } \\
\text { gramática e da } \\
\text { compreensão, } \\
\text { hesitações na fala, } \\
\text { repetições de palavras e } \\
\text { dificuldade da escolha } \\
\text { lexical }\end{array}$ & $\begin{array}{c}\text { Educação de pacientes } \\
\text { e cuidadores }\end{array}$ & $\begin{array}{c}\text { A fluência verbal é } \\
\text { comprometida nesses } \\
\text { pacientes }\end{array}$ \\
\hline
\end{tabular}

Fonte: Silva NS, et al., 2020. Baseado em Sierra-Peláez S e Villegas-Lanau CA, 2015.

A DFT variante comportamental (DFTvc) é a forma mais prevalente dessa síndrome, correspondendo por cerca de $90 \%$ dos casos e, geralmente caracterizada por alterações comportamentais progressivas (UCEDO DM, et al., 2017; VALENTE ES, 2019).

Em estudo de Valente ES (2018) acerca das formas de DFT, descobriram-se que as disfunções mais prevalentes dessa forma são apatia, descuido com a aparência pessoal, impulsividade, irritabilidade, aumento do apetite, perda de interesse por atividades antes realizadas e atitudes motoras estereotipadas. Além disso, os pacientes com DFTvc apresentaram conversação normal, enquanto os demais manifestaram pelo menos uma alteração da linguagem, como ecolalia, afasias anômicas, como a não fluente mista ou a de Wernicke (CUETOS F, et al., 2010; VALENTE ES, 2018). 
A forma semântica ou temporal da DFT é caracterizada por afasia nominal e por distúrbios do comportamento, em decorrência da neurodegeneração assimétrica dos lobos afetados. Nos exames neurológicos, as habilidades visuoespaciais e a função executiva permanecem praticamente intocáveis (RIVAS-NIETO JC, 2014).

As alterações comportamentais que podem advir nesse subtipo de DFT, são diferentes das manifestadas na DFTvc. Nesse caso, os pacientes costumam manter atividades únicas, como pintura, por longos períodos, além de poder manifestar comportamentos hipocondríacos ou histriônicos (VALENTE ES, 2018; RODRÍGUEZ-LEYVA I, 2019).

Conforme Rivas-Nieto JC (2014), a afasia progressiva primária (APP) é outra apresentação da DFT, com maior prevalência em mulheres. Caracteriza-se por alterações importantes da fala, como diminuição do tamanho das orações, parafasias fonológicas, mudanças na velocidade da linguagem e erros de gramática, com sintaxe preservada. Existe ainda uma relativa conservação da inteligência não verbal, da compreensão e da memória, que permitem a classificação dessa variante. O primeiro sinal da APP é a anomia, que pode apresentar um déficit linguístico progressivo, evoluindo para mutismo completo (RIVAS-NIETO JC, 2014; UCEDO DM, et al., 2017).

Existem associações da DFT com outras enfermidades, como a Esclerose Lateral Amiotrófica (ELA), conjuntas em até $15 \%$ dos casos (MARTINS J, et al., 2013). No estudo de Lillo P, et al. (2014) acerca da sobreposição da DFT e ELA, demonstrou que na DFTvc, o início costuma ser insidioso e o paciente não apresenta consciência do declínio neurofuncional. As alterações comportamentais típicas dessa doença, como distúrbios da personalidade e da conduta social, refletem lesões nas áreas orbitais e mesiais dos lobos frontais (LILLO P, et al., 2014; SCHLINDWEIN-ZANINI R, et al., 2016).

Em uma descrição clínica, neuropsicológica e de neuroimagem de 21 pacientes com DFT, Rivas-Nieto JC (2014) expôs que alterações importantes na DFTvc incluem: desinibição social, transtornos da personalidade, com evolução para atitudes sociopatas e perdas da higiene pessoal e do controle dos esfíncteres. $O$ estudo demonstra que a neuroimagem permite boa avaliação inicial e prognóstica desses pacientes, mostrando na fase inicial alterações atróficas e de hipoperfusão nos lobos frontais e insular. Com o avanço neurodegenerativo, a atrofia cortical estende-se progressivamente para regiões temporoparietais (RIVASNIETO JC, 2014; SANTOS KP, et al., 2015).

Facilitando a abordagem e reconhecimento dos pacientes com DFTvc, desenvolveu-se algumas escalas, como a de Raschkovsky, que em consonância com uma história clínica detalhada e exames imagiológicos, pode contribuir para a elucidação diagnóstica da DFTvc (LILLO P, et al., 2014).

Essa escala avalia uma série de sintomas em pacientes com suspeita de demências, como desinibição social, apatia, perda precoce da simpatia ou empatia, comportamento perseverante ou estereotipado, mudanças da dieta e do perfil comportamental do paciente, e, perfil neuropsicológico. Após a avaliação, o paciente pode ser classificado como DFTvc possível, DFTvc provável, DFTvc comprovada ou apresentar critérios de exclusão para esse tipo de síndrome demencial (CUSTÓDIO N, et al., 2018; LILLO P, et al., 2014).

\section{DISCUSSÃO}

\section{Tratamento multidisciplinar da Demência Frontotemporal}

Não existe terapêutica farmacológica bem estabelecida para tratar as disfunções neuropsiquiátricas da DFT, principalmente em relação à DFTvc, cujas modificações interferem negativamente no cotidiano do portador e daqueles que convivem de maneira próxima. Algumas classes de medicamentos, a exemplo dos neurolépticos, antidepressivos, anticonvulsivantes e agonistas dopaminérgicos costumam ser usados, mas os estudos que abordam essa temática carecem de rigor metodológico adequado. Todavia, algumas formas de tratamento não convencionais e multidisciplinares têm se destacado nessa perspectiva, e, podem funcionar como boas alternativas no tratamento dessa síndrome demencial (O'CONNOR CM, et al., 2013). 
Com base nessa terapêutica inconvencional, O'Connor CM, et al. (2013) descreveram que existem três formas mais comuns de abordagem não-farmacológica para a DFT. A primeira, pautada no paciente e na melhora do seu quadro. A segunda, com foco no cuidador, visando o progresso da sua qualidade de vida. E, por fim, uma terapêutica voltada para ambos. Embora haja essa divisão, a atenção profissional multidisciplinar aos pacientes com DFT ainda é pouco utilizada, ao contrário do que acontece, por exemplo, na DA (O'CONNOR CM, et al., 2013).

Antes da instituição de uma abordagem multidisciplinar, entretanto, é necessário avaliar todo o quadro clínico do paciente. Vários sintomas neuropsiquiátricos presentes nesses casos, por exemplo, podem ser resultantes de outras comorbidades comuns nos idosos, como infecções, descompensação metabólica, desidratação, efeitos colaterais indesejados de drogas, fraturas, traumatismos cranianos e outros. Assim que essas outras possíveis causas forem afastadas do raciocínio clínico é possível iniciar a terapêutica, que deve ser pautada em métodos diversificados (LIMA-SILVA TB, et al., 2013).

Além disso, recomenda-se que sejam desenvolvidas alterações no ambiente em que esses indivíduos vivem, tornando-os mais agradáveis, e nos seus hábitos de vida. Sugere-se, que eles passem a praticar atividades físicas leves, como caminhadas e/ou atividades hídricas de baixo impacto, e, utilizem a música como forma de terapia. O uso da musicoterapia costuma ser aplicado para o tratamento de sintomas comportamentais e psicológicos da demência. Durante momentos de higiene pessoal, banhos e troca de roupas, nos quais o portador pode se apresentar agitado e agressivo, a música funciona diminuindo os acessos de ansiedade e facilitando o cuidado por familiares. Alguns estudos mostraram redução de até 50\% dessa sintomatologia, graças às intervenções musicais progressivas (LIMA-SILVA TB, et al., 2013; O'CONNOR CM, et al., 2013).

Com relação aos exercícios físicos, existem importantes similitudes entre essa prática e a diminuição dos sintomas neuropsiquiátricos e comportamentais. O hábito de executar caminhadas matutinas está associado a menores disfunções no ritmo circadiano dos pacientes e, por consequência, resulta em menos despertares noturnos. O comportamento motor aberrante dos idosos com DFT parece também melhorar com as atividades. Quando a deambulação é estimulada em um ambiente seguro, como o quintal da casa, ou em uma praça próxima à residência, as tentativas de saídas furtivas dos pacientes acabam diminuindo (BARTON C, et al., 2016).

Devido aos déficits relacionados aos lobos parietais e temporais, os pacientes tendem a desenvolver uma capacidade limitada de compreensão e, por conseguinte, episódios de ansiedade e irritabilidade referentes a modificações ambientais. Para evitar isso, é importante que o acesso a itens cotidianos geradores de estresse, como cartões de crédito e contas bancárias, seja limitado. Ademais, é interessante que tais indivíduos realizem acompanhamento psicológico, de modo a simplificar, com qualidade, a vida do indivíduo com DFT e reduzir esses fenômenos desgastantes (BARTON C, et al., 2016).

Além disso, como tais pacientes costumam apresentar desregulação do ciclo sono-vigília, uma alternativa viável é o fechamento de portas, janelas ou espaços por onde a luz possa entrar durante o dia, por um determinado período, no intuito de reduzir esses distúrbios e não perturbar o ritmo circadiano do doente (LANCHO MCP, BERCIANOS SG, 2018).

Outrossim, como os pacientes com DFT tendem a ter uma menor sociabilidade, a convivência em espaços com muitas pessoas pode ser incômoda, tanto para o paciente, quanto para àqueles que estão ao seu redor. Nesses cenários, algumas atividades compulsivas podem surgir, como a necessidade incessante de tocar nas outras pessoas, ou ainda, vocalizações constantes. Uma forma de burlar esses fenômenos é oferecer objetos ou alimentos agradáveis ao paciente, como bolas de apertar e pirulitos (BARTON C, et al., 2016).

Ademais, ainda nesse contexto, alguns idosos podem apresentar um comportamento sexual incontrolável. Obviamente, os pacientes necessitam de um acompanhamento psicológico diferenciado nesses casos, mas uma estratégia não convencional que pode ser utilizada é o oferecimento de um boneco de pelúcia de tamanho real, para que o doente possa acariciar e aliviar essa sexualização. Todavia, essas práticas devem ser aplicadas de forma individual e sempre devem ter a supervisão do profissional e do cuidador (BARTON C, et al., 2016). 
Outro ponto importante a ser manejado por profissionais de múltiplas áreas terapêuticas é a linguagem. Assim como a avaliação de alguns elementos linguísticos ajudam na classificação da DFT, ela pode fornecer dados qualitativos que permitem um melhor monitoramento do paciente e das suas possibilidades de tratamento (MARTíNEZ A, et al., 2015).

Andrade-Calderón $P$, et al. (2015), relataram que a terapia do discurso, a partir de intervenções fonoaudiológicas, possui potencial de melhorar alguns aspectos clínicos dos pacientes. Foram descritas melhoras no ritmo, prosódia, fluência e conteúdo da fala espontânea, associadas com avanços cognitivos e na tomada de decisões, e diminuição de sintomas neuropsiquiátricos, em decorrência da aplicação dessa técnica (ANDRADE-CALDERÓN P, et al., 2015; MARTíNEZ A, et al., 2015).

O tratamento multidisciplinar também deve ser estendido aos familiares e cuidadores das pessoas com DFT. Sabe-se que esses indivíduos, em geral, convivem com situações desgastantes e de grande envolvimento emocional, psicológico e físico, que acabam prejudicando a qualidade de vida, podendo provocar a síndrome da exaustão do cuidador, semelhante à síndrome de Burnout, caracterizada por esgotamento emocional, mental e físico (MARIANO LI, 2018).

Os estudos de Mariano LI (2018), por exemplo, discutiram que a falta de conhecimento sobre a DFT faz com que os cuidadores sofram mais episódios da síndrome da exaustão do cuidador e estes ocorrem com maior gravidade. Por isso, foram idealizadas conferências específicas sobre a doença, seu curso clínico e com algumas dicas para facilitar o processo de cuidado (MARIANO LI, 2018).

Uma outra alternativa na terapêutica envolvendo os cuidadores de indivíduos com DFT é a utilização dos recursos do programa de saúde do cuidador de Alzheimer. Dentre eles, destacam-se cursos sobre a segurança do cuidado em casa, resolução de empecilhos, redução do estresse e promoção de saúde, que possuem resultados positivos sobre as manifestações comportamentais dos pacientes enfermos e auxiliam na melhoria da qualidade de vida dos cuidadores (GITLIN L, et al., 2012; CECCHINI MA, 2017; KOLANOWSKI A, et al., 2017; MARIANO LI, 2018).

Em um estudo randomizado por Hayden LJ, et al. (2012) estruturaram uma plataforma digital voltada para os cuidadores dos pacientes com demência. Nesse serviço estavam disponíveis uma série de vídeos explicando o processo fisiopatológico da demência e seus sintomas decorrentes (HAYDEN LJ, et al., 2012).

Além disso, o site possuía salas de bate-papos semanais com diversos especialistas, como geriatras, psiquiatras, psicólogos e sociólogos, que ajudaram os familiares a entender um pouco mais sobre a doença, aliviando sua carga emocional e psíquica, e facilitando o manejo dos pacientes e o autocuidado. Embora essa seja uma intervenção interessante, eficaz e diferente, ela ainda não é acessível a maior parte dos cuidadores, mas constitui-se uma alternativa louvável para a melhoria da saúde do familiar e para lidar melhor com o luto e a sensação de culpa que possa vir a existir (HAYDEN LJ, et al., 2012).

Com base na terapêutica para ambos os grupos, uma das principais estratégias de manejo é o Programa de Atividades Personalizadas (PAP). O PAP é uma intervenção comunitária baseada em terapia ocupacional, projetada para reduzir os sintomas comportamentais e psicológicos da DFT, prescrevendo atividades personalizadas, em conjunto com a educação do familiar ou cuidador (GITLIN L, et al., 2012).

Esse modelo foi descrito por Gitlin L, et al. (2012) e apresenta resultados bastante satisfatórios. Ao contrário de outros modelos de tratamento não farmacológico, o PAP envolve a estruturação de atividades baseadas em tarefas únicas, para auxiliar com deficiências no funcionamento executivo da DFT. Além disso, para um bom desempenho do paciente, é necessário um compromisso do cuidador para com ele e um ambiente adequado para a execução da técnica (GITLIN L, et al., 2012).

Em relação aos resultados, foi verificado que após a intervenção com o PAP, os pacientes com DFT acabavam se envolvendo com mais atividades, de forma organizada e efetiva. Isso permitiu que sintomas típicos, como a agitação, sofressem diminuições significativas. Por conta desse maior envolvimento e ocupação temporal dos pacientes, os cuidadores desfrutavam de maior liberdade e tempo para cuidar de si mesmos, melhorando a qualidade de vida de uma forma geral (O'CONNOR CM, et al., 2013). 


\section{Perspectivas futuras no tratamento de Demência Frontotemporal}

A perspectiva futura é que as terapias abranjam o paciente com DFT de modo holístico, para que este quadro não permaneça tão desafiador nos aspectos de manipulação dos conhecimentos etiopatogênicos e a sua aplicação nas vertentes de abordagem diagnóstica e terapêutica. Entende-se que o espectro biopsicossocial desta doença perpassa por várias dificuldades, o que influencia diretamente na vida do paciente, bem como dos familiares e cuidadores. Frente a isso, depreende-se que serão necessários investimentos das diversas áreas para questões como o tratamento farmacológico e não farmacológico. Entretanto, não apenas deve-se focar no tratamento dessa enfermidade (BRUCKI SMD, et al., 2011; LILLO P, et al., 2014).

É necessário haver um diagnóstico apropriado e legítimo para que assim seja possível proceder de forma mais rápida e acurada à DFT, partindo de métodos como a escala de Raschkovsky para inspiração nesta área. Dessa maneira, sugere-se que sejam delineadas pesquisas que sugiram, ou até mesmo, estabeleçam as melhores metodologias diagnósticas a fim de se ter uma abordagem inicial mais eficiente e rápida para que assim, a condução do paciente seja mais direcionada para a doença. A partir disso, será possível, no futuro, a demonstração e a comprovação científica de novas técnicas de controle terapêutico no processo de saúde-doença da DFT, de forma mais efetiva e apropriada, contemplando ainda, os cuidadores e os familiares (BRUCKI SMD, et al., 2011; LILLO P, et al., 2014).

\section{CONSIDERAÇÕES FINAIS}

A DFT é uma doença neurodegenerativa de grande impacto para o paciente e sua família, necessitando de diagnóstico breve e adequada resposta terapêutica. Não existe uma terapia farmacológica específica para as disfunções neuropsiquiátricas, todavia, há terapia de suporte que devem ser individualizadas. A estratégia multidisciplinar da DFT pode ser direcionada para o paciente, para o cuidador ou ambos. Alguns pontos terapêuticos não farmacológicos multidisciplinares são promissores proporcionando espaço neutro para as situações e sintomas neuropsiquiátricos. Por fim, as intervenções em terapia ocupacional associada a educação do familiar ou do cuidador auxiliam no manejo da DFT.

\section{REFERÊNCIAS}

1. ANDRADE-CALDERON P, et al. Positive Impact of Speech Therapy In Progressive Non-Fluent Aphasia. Act. Colom. Psicol., Bogotá, julho 2005; 18(2): 101-114.

2. BARTON C, et al. Non-pharmacological management of behavioral symptoms in frontotemporal and other dementias. Current neurology and neuroscience reports, 2016; 16(2): 14.

3. BRUCKI SMD, et al. Demências-enfoque multidisciplinar: das bases fisiopatológicas ao diagnóstico e tratamento. In: Demências-enfoque multidisciplinar: das bases fisiopatológicas ao diagnóstico e tratamento. 2011. p. 407-407.

4. CECCHINI MA. Teste de memória integrativa: comparação de desempenho entre demência frontotemporal variante comportamental e doença de Alzheimer. 2017; 153p. Tese de Doutorado. Universidade de São Paulo.

5. CORDEIRO Q, et al. Genética das demências. Arquivos Médicos dos Hospitais e da Faculdade de Ciências Médicas da Santa Casa de São Paulo, 2018; 53(1): 24-30.

6. CUETOS F, et al. Tipos de trastornos anómicos en las afasias. Revista de logopedia, foniatría y audiología, 2010; 30(1): 16-22.

7. CUSTÓDIO N, et al. Análisis comparativo de las características neuropsicológicas de pacientes con demencia frontotemporal, variante conductual y enfermedad de Alzheimer. Revista neuropsiquiatria. 2012; 75(4): 120-128.

8. CUSTÓDIO N, et al. Evolución histórica del concepto y criterios actuales para el diagnóstico de demencia. Revista Neuropsiquiatria. outubro 2018; 81(4): 235-249.

9. FORNARI LHT, et al. As diversas faces da síndrome demencial: como diagnosticar clinicamente? Scientia medica. 2010; 20(2): 185-193.

10. FREIRE R. Uma abordagem matemática para auxiliar o diagnóstico de demências: tratando incertezas e quantificando processos. 2014. Tese de Doutorado. Universidade de São Paulo.

11. GITLIN L, et al. Nonpharmacologic management of behavioral symptoms in dementia. Jama. 2012; 308(19): 20202029.

12. HAYDEN LJ, et al. The use of Internet technology for psychoeducation and support with dementia caregivers. Psychological Services. 2012; 9(2): 215-218.

13. KOLANOWSKI, A et al. Determinants of behavioral and psychological symptoms of dementia: A scoping review of the evidence. Nursing outlook. 2017; 65(5): 515-529. 
14. LANCHO MCP; BERCIANOS SG. Demencia frontotemporal: fronteras diagnósticas. International Journal Of Developmental And Educational Psychology. Revista Infad de Psicología. maio 2018; 4(1): 67-76.

15. LILLO P, et al. Manifestaciones neuropsiquiátricas y cognitivas en demencia frontotemporal y esclerosis lateral amiotrófica: dos polos de una entidad común. Rev. méd. Chile, Santiago, julho 2014; 142(7): 867-879.

16. LILLO P, LEYTON C. Demencia frontotemporal, cómo ha resurgido su diagnóstico. Revista Médica Clínica Las Condes, 2016; 27(3): 309-318.

17. LIMA-SILVA TB, et al. Functional profile of patients with behavioral variant frontotemporal dementia (bvFTD) compared to patients with Alzheimer's disease and normal controls. Dementia \& neuropsychologia, 2013; 7(1): 96103.

18. MARIANO LI. Impulsividade e controle inibitório: avaliação cognitiva, comportamental e diagnóstica na demência frontotemporal. 2017. 129p. Dissertação (Mestrado) - Curso de Neurociências, Instituto de Ciências Biológicas, Universidade Federal de Minas Gerais, Belo Horizonte, 2017. Disponível em: http://hdl.handle.net/1843/BUOSB3VN95. Acesso em: 04 jun. 2020.

19. MARTÍNEZ A, et al. Valoración de prosodia espontánea afectiva y análisis de discurso en pacientes con esquizofrenia y demencia frontotemporal (DFT) variante lingüística. Rev. Colomb. Psiquiatr., Bogotá, janeiro 2015; 44(1): 13-19.

20. MARTINS J, et al. Demência frontotemporal associada à esclerose lateral amiotrófica: relato de caso. Geriatrics, Gerontology and Aging, 2013; 7(4): 318-321.

21. O'CONNOR CM, et al. Enhancement of carer skills and patient function in the non-pharmacological management of frontotemporal dementia (FTD): a call for randomised controlled studies. Dement Neuropsychol, Austrália, maio 2013; 2(7): 143-150.

22. PANTOJA G, et al. Enfermedad neurodegenerativa: demencia frontotemporal. Sanidad Militar, 2020; 76(1): 39-40.

23. RIVAS-NIETO JC. Frontotemporal dementia: clinical, neuropsychological, and neuroimaging description. Colomb. Med., Cali, julho 2014; 45(3): 122-126.

24. RODRÍGUEZ-LEYVA I et al. Demencia frontotemporal: revisión y nuestropunto de vista. Revista Mexicana de Neurociencia, [S.L.], outubro 2019; 19(6): 20-31.

25. SANTOS KP, et al. A fluência na afasia progressiva primária logopênica. Audiol., Commun. Res., São Paulo, setembro 2015; 20(3): 285-291.

26. SCHLINDWEIN-ZANINI R, et al. Aspectos neuropsicológicos da Esclerose Lateral Amiotrófica: relato de caso. Arquivos Catarinenses de Medicina, 2016; 44(1): 62-70.

27. SIERRA-PELÁEZ S, VILLEGAS-LANAU, CA. Microquimerismos en la enfermedad de Alzheimer y la demencia frontotemporal. Medicina y Laboratorio, 2015; 21(7-8): 333-348.

28. SOUZA MF. Variante comportamental da Degeneração Lobar Frontotemporal: padrões neuropsicológicos e discursivos. Trabalho de conclusão de especialização (Especialização em Neuropsicologia) - Instituto de Psicologia. Universidade Federal do Rio Grande do Sul, Porta Alegre, 2011; 47p.

29. UCEDO, DM; et al. A linguagem na Demência Frontotemporal: uma análise à luz da Neurolinguística EnunciativoDiscursiva. CoDAS, São Paulo, 2017; 29(4):1-6.

30. VALENTE ES. Fenocópias de demência frontotemporal: revisão sistemática. 2018. 48 f. Monografia (Doutorado) Curso de Neurociências, Instituto de Ciências Biológicas, Universidade Federal de Minas Gerais, Belo Horizonte, 2018. 